\title{
CAPITULO 79
}

\section{PROMOÇÃO E EDUCAÇÃO EM SAÚDE FRENTE AO IMPACTO DAS QUEIMADURAS EM CRIANÇAS}

\section{DOI 10.4322/978-65-995353-2-1.c79}

Tatiana Indiana da Silva ${ }^{1}$, Ana Luiza Evangelista da Silva ${ }^{2}$, Ellyan Victor Ferreira dos Santos $^{3}$, Rosa Raquel Pinto Guedes Marques ${ }^{4}$, Ísis Passos Ferreira ${ }^{5}$,Emanuelly Ferreira da Silva ${ }^{6}$, Matheus Emanuel Souza Araújo Vieira ${ }^{7}$, Maria Fernanda da Silva ${ }^{8}$, karolayne Carvalho da Silva ${ }^{9}$, Roberta Luciana do Nascimento Godone ${ }^{10}$

${ }^{1}$ Acadêmica em Enfermagem do Centro Universitário Mauricio de Nassau/Caruaru-PE, (tatianaindiana10@gmail.com)

${ }^{2}$ Acadêmica em Enfermagem do Centro Universitário Mauricio de Nassau/Caruaru-PE, (luizaana10@hotmail.com)

${ }^{3}$ Acadêmico em Enfermagem do Centro Universitário Mauricio de Nassau/Caruaru-PE, (ellyansantos12@gmail.com)

${ }^{4}$ Acadêmico em Odontologia da Universidade Federal Fluminense Campus de Nova Friburgo, (rosa.raquel.marques@gmail.com)

${ }^{5}$ Acadêmica em Odontologia do Centro Universitário Fametro, Manaus-AM, (isisfpassos@gmail.com)

${ }^{6}$ Acadêmica em Enfermagem do Centro Universitário Maurício de Nassau/Caruaru-PE, (emanuelly.f.ef@gmail.com)

${ }^{7}$ Acadêmico em Enfermagem do Centro Universitário Maurício de Nassau/Caruaru-PE, (matheusvieira.7@outlook.com)

${ }^{8}$ Acadêmica em Fisioterapia do Centro Universitário Mauricio de Nassau/Caruaru-PE, (fernanda16062001@gmail.com)

${ }^{9}$ Acadêmica em Enfermagem do Centro Universitário Maurício de Nassau/Caruaru-PE, (Karol66carvalho@gmail.com)

${ }^{10}$ Universidade Federal Rural de Pernambuco, Recife, PE. Phd em biologia Aplicada a saúde UFPE (robertagodone@hotmail.com) Pesquisa e Extensão 


\begin{abstract}
Resumo
Introdução: A queimadura na infância é um problema de saúde pública grave, que incide principalmente nos países em desenvolvimento, como o Brasil. A decorrência de acidentes com crianças é um fator preocupante, por sua imaturidade física e cognitiva, portanto, elas possuem altas chances de cair e se machucarem, reforçando a supervisão dos responsáveis, para a sua proteção. Objetivo: Revisar a Literatura acerca da promoção e prevenção de saúde frente às queimaduras infantis. Método: Trata-se uma revisão bibliográfica, onde o levantamento foi realizado nas bases de dados da LILACS, Scielo e PubMed, por meio do operador booleano AND, sendo coletados artigos entre 2016 e 2020, utilizando os Descristores (DeSC): Educação em Saúde; prevenção de queimaduras; equipe multidisciplinar. Resultados: A educação em saúde no combate a acidentes por queimaduras é feita pelos enfermeiros durante as suas consultas e visitas domiciliares. As estratégias educacionais alcançam pais e cuidadores, resultando na conscientização dos mesmos e mudanças no ambiente a fim de torná-lo mais seguro. A equipe multidisciplinar também atua, trabalhando de forma conjunta, dividindo a atenção e cuidado ao paciente. Pode-se citar a exemplo, o trabalho da odontologia e fisioterapia no cenário de queimados, já que esses pacientes apresentam risco de desenvolvimento de problemas físicos, locomotores e dificuldade de se realizar a higiene bucal. O que mostra o tamanho da importância de orientação aos responsáveis e aos enfermeiros da saúde da criança, durante o período de internação. Conclusão: A prevenção das queimaduras em crianças é de responsabilidade dos adultos, sejam seus pais ou aqueles responsáveis pelos cuidados delas. Todos devem receber formação sobre prevenção de acidentes e, acima de tudo, das queimaduras. Essa formação deve ser destinada a evitar fatores de risco de queimaduras em crianças, levando em conta os diferentes ambientes em que estes ocorrem.
\end{abstract}

Palavras-chave: Educação em saúde; Equipe multidisciplinar; Prevenção de queimaduras. Área Temática: Temas livres.

E-mail do autor principal: tatianaindiana10@gmail.com

\title{
1 INTRODUÇÃO
}

A prática da educação em saúde é uma das atividades que requer grande atenção, por sua magnitude, por isso ela deve ser entendida como uma importante vertente à prevenção. Os acidentes na infância são diversos, elevando a necessidade de preveni-los, principalmente o que ocorrem por queimaduras. A Organização Mundial de Saúde (OMS) define como acidente "um acontecimento independente da vontade humana", contudo, o padrão usual que o compreende como inevitável e imprevisível vem sendo transformado e uma nova concepção conceitua o acidente como um episódio previsível, capaz de gerar lesões ou até o óbito. (BATALHA et al.,

E - book Tripé do Ensino Superior: Ensino, Pesquisa e Extensão 
2016) Os acidentes domésticos na infância (ADI), embora subestimados, caracterizam-se como um relevante problema de saúde no mundo, devido à sua potencial gravidade (SILVA et al., 2017).

Nesse sentido, ocorrência de ADI possui relação direta com o comportamento familiar e sociedade, estando relacionada aos hábitos de vida, condições educacionais, econômicas, sociais e culturais, associada à idade específica das crianças, somada à curiosidade e desejo de aprender (MOITA et al., 2018). A queimadura na infância é um problema de saúde pública grave que incide principalmente nos países em desenvolvimento como o Brasil. A decorrência de acidentes com crianças é um fator preocupante, por sua imaturidade física e cognitiva elas possuem altas chances de cair e se machucarem, reforçando a supervisão dos responsáveis, para sua proteção.

\section{MÉTODO}

Este trabalho se trata de uma revisão bibliográfica, onde o levantamento foi realizado nas bases de dados da LILACS, Scielo e PubMed, através do operador booleano AND, sendo coletados artigos entre 2016 e 2021. Foi utilizado os Descristores (DeSC) e estratégia PPC (população, problema e controle), no qual corresponde: P:Crianças, P: Queimaduras, C: Promoção e educação. Com isso, foi formulada a pergunta norteadora: "Quais as evidências da literatura científica contextualizam a respeito da promoção em saúde frente ao impacto das queimaduras em crianças?”. Foram incluídos artigos completos, com aderência ao estudo, nos idiomas português e inglês. Foram excluídos artigos duplicados, anais de congressos, estudos sem aderência e fora dos anos estabelecidos e dos idiomas supracitados. Foram analisados 15 artigos completos e após análise criteriosa, foram selecionados 11 artigos que compuseram a amostra final.

\section{RESULTADOS E DISCUSSÃO}

A educação de saúde no combate a acidentes por queimaduras é feita na Atenção Primária pelos enfermeiros durante as consultas e visitas domiciliares. Isso é feito através de medidas educativas, como banners, simulações criativas, palestras, e diálogos com os pais, onde

\section{E - book Tripé do Ensino Superior: Ensino,} Pesquisa e Extensão 
através disso, eles poderão desenvolver papel muito importante na promoção de saúde. Uma das estratégias possíveis é modificar o ambiente de acordo com a idade da criança, sempre levantar hipóteses do que pode acontecer e assim, afastá-la dos locais mais perigosos, pois, quanto mais baixa a faixa etária da criança, mais chances de acidentes. As crianças menores de três anos são mais suscetíveis às queimaduras, e isso é explicado pela sua curiosidade natural, elas são impulsivas e não possuem discernimento para avaliar os perigos. O cuidado com a exposição ao sol também é imprescindível, pois as crianças ficam em áreas abertas de 16 a 60 minutos, um tempo suficiente para causar queimaduras solares (STANGANELLI et al., 2020).

É necessário ter atenção aos objetos fora dos domicílios, que podem com o aquecimento do sol causar essas lesões, como o metal quente. Devido a isso, a área mais afetada são os pés, por frequentemente estarem descalças ou com sapatos inadequados. Além disso, é importante que a temperatura de brinquedos seja verificada antes de expor a criança e, assim, será possível ser evitada lesões. Como também, limitar a exposição ao sol entre o horário de dez da manhã a dezesseis horas da tarde, reduzindo o risco de ocorrências solares. Podendo ocasionar lesões de grau 1, menos avançado, até o grau 3, onde compromete boa parte do tecido (CHOI et al., 2019). (Tabela 1).

Tabela 1. Características acerca dos graus, a superfície e os agentes etiológicos de queimaduras em crianças internadas no Centro de Tratamento de Queimados do Hospital Metropolitano de Urgência e Emergência, Ananindeua,

\begin{tabular}{|lcc|}
\hline \multicolumn{1}{|c}{ Características } & $\mathbf{n}$ & $\%$ \\
\hline Grau da queimadura & & \\
\hline $1^{\circ}$ Grau & 34 & 9,69 \\
\hline $2^{\circ}$ Grau & 285 & 81,20 \\
\hline $3^{\circ}$ Grau & 32 & 9,12 \\
\hline Superficie Corporal Queimada & \\
\hline Pequeno queimado & 141 & 46,38 \\
\hline Médio queimado & 106 & 34,87 \\
\hline Grande queimado & 57 & 18,75 \\
\hline Agente etiológico & & \\
\hline Escaldadura & 152 & 50 \\
\hline Substância inflamável & 44 & 14,50 \\
\hline Contato & 89 & 3,30 \\
\hline Radiaçå solar & 10 & 1,62 \\
\hline Eletricidade & 5 & 29,3 \\
\hline
\end{tabular}

Fonte: Tabela adaptada da Revista Brasileira de Queimaduras, 2015.

http://www.rbqueimaduras.com.br/details/265/pt-BR/caracterizacao-das-criancas-vitimas-de-queimaduras-emhospital-de-referencia-na-regiao-amazonica 
Portanto, há necessidade de novas campanhas para se evitar queimaduras na infância, como também se faz necessário que os pediatras, nos postos de saúde e consultórios, façam um pequeno alerta aos pais das maneiras habituais de prevenção aos acidentes domésticos, visto que, estatisticamente, a maior incidência de acidentes ocorre em ambiente domiciliar. Estudos relatam que boa parte das vítimas, é a população infantil.

Figura 1. Faixa etária de pacientes queimados atendidos no periodo de 2013 a 2018 no hospital infantil da Serra Catarinense, 2019.

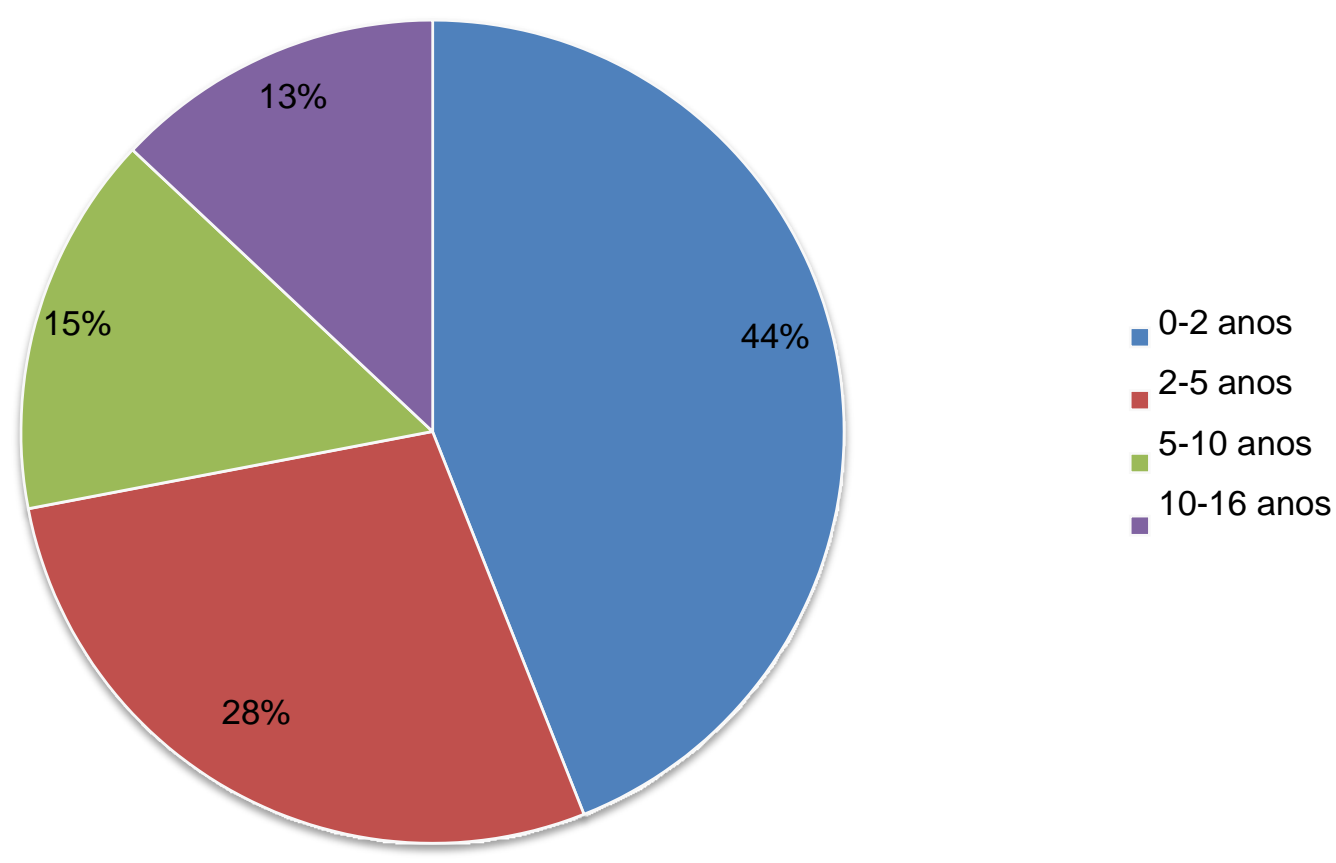

Fonte: Figura adaptada da Revista Brasileira de Queimaduras

< http://www.rbqueimaduras.com.br/details/467/pt-BR/perfil-epidemiologico-das-criancas-vitimas-dequeimaduras-em-um-hospital-infantil-da-serra-catarinense $<$

O grupo infantil é o mais atingido por queimaduras, e o profissional da enfermagem é o mais capacitado como mediador na Atenção Básica, por possuir grande aproximação e confiança da população. Assim, fica sob sua incumbência a orientação e educação aos pais sobre os acidentes domésticos na infância e formas de prevenção (PASSOS \& SANTOS, 2016). Pesquisa e Extensão 
Fortalecer os conhecimentos junto aos pais e familiares, sobre a magnitude da prevenção dos Acidentes doméstico na infância (ADI) é de fundamental importância. Sobretudo, expor apenas as formas de prevenções de determinados agentes causadores de acidentes não são o suficiente, é necessário compreender a família como um todo; condições socioeconômicas, demográficas e culturais, condições de moradia e familiares impactam direta ou indiretamente na prevenção dos ADI. Diante do conhecimento desses fatores, é possível desenvolver estratégias direcionadas, evidenciando os fatores de riscos, as medidas de segurança e finalmente, reduzir a incidência de ADI (LIMA et al., 2019). Na Atenção Primária, desenvolver estratégias educacionais para o alcance de pais e cuidadores, pode resultar na conscientização dos mesmos e mudanças no ambiente a fim de torná-lo mais seguro (BRITO et al., 2017). Esses profissionais devem estar em constante atualização dos conhecimentos, uma vez que dúvidas possam surgir (MARGOTTI et al., 2018).

Ressalta-se que a presença de adultos na hora dos acidentes nem sempre é fator protetivo para a ocorrência deles. Por isso, é necessário que além das orientações de prevenção, a educação sobre como agir diante de determinadas situações façam parte do planejamento. $\mathrm{O}$ eficiente conhecimento de pais e cuidadores sobre primeiros socorros e a correta maneira de agir, podem resultar na redução de danos (SANCHES et al., 2016).

A equipe multidisciplinar também faz parte da educação em saúde, trabalhando de forma conjunta, dividindo a atenção e cuidado ao paciente. Pode-se citar a exemplo, o trabalho da odontologia no cenário de queimados, já que esses pacientes apresentam risco de desenvolvimento de problemas dentários, como a cárie e a doença periodontal, devido à dificuldade de uma adequada higiene bucal. A fisioterapia, por sua vez, atua no tratamento dos pacientes queimados com objetivo de fazê-lo retornar as suas atividades cotidianas, melhorando sua qualidade de vida. Para isso, a reabilitação do paciente tem como foco: manutenção da força muscular e da Amplitude de Movimento (ADM), diminuição do risco de infecções, obtenção de deambulação independente, mínima formação de cicatrizes, prevenção de contraturas e deformidades, aumento da capacidade aeróbia e restauração da resistência cardiovascular. Por outro lado, por atender o público infantil, esses pacientes sentem-se expostos a pessoas fora de seu vínculo afetivo, em um espaço diferente do que é costumado. Precisando-se ter enquanto profissional, paciência, motivação e construção afetiva a fim de passar o máximo de segurança à criança. Porque, infelizmente, o tratamento fisioterapêutico é doloroso, e mostrar o quanto se importa com o pequeno(a), traz conforto também a familia neste momento de preocupação, 
além de considerar que esse trauma psicológico (queimaduras graves) é capaz de afetar o progresso do tratamento.

Diante do exposto, mostra-se o tamanho da importância de orientação aos responsáveis e aos enfermeiros da criança, durante o período de internação. Além disso, alternativas de tratamento e métodos de promoção de saúde bucal, devem ser estudados especificamente pela comunidade odontológica. Se torna indispensável a presnça da equipe multiprofissional, para verificar as características da queimadura, bem como para decidir a melhor conduta terapêutica, objetivando o alívio do sofrimento do paciente durante os procedimentos indicados no tratamento. Tendo em vista a dimensão da queimadura, para o controle e alívio da dor são utilizados tratamentos farmacológicos e não farmacológicos (SCAPIN S et al., 2017). A exemplo do recurso não farmacológico utliza-se a realidade virtual (RV), classificada como analgesia distrativa, que faz uma simulação de um cenário real, por meio de uma interface indivíduo-máquina o paciente corresponde ao que é simulado por meio da potencial capacidade de interação e imersão produzidas, de forma que a atenção seja desviada do ambiente real para o ambiente virtual com a exposição de animações tridimensionais, em tempo real, promovendo assim, a interação e, consequentemente, distração durante o atendimento, sendo aplicada principalmente nas condutas que geram um estímulo maior à dor (CARICCHIO MBM, 2017; SCAPIN S et al., 2017). Estudos relatam resultados favoráveis desse tratamento em pacientes queimados, resultando na diminuição da percepção de intensidade dolorosa e ansiedade (FARIAS MB et al., 2019). Com enfoque da neurociência moderna, para compreender as respostas cerebrais de crianças às situações por elas vivenciadas. Nesse âmbito, a RV é considerada um meio facilitador promissor de tratamento de várias afecções (CARICCHIO MBM, 2017).

\section{CONCLUSÃO}

A prevenção das queimaduras em crianças é responsabilidade dos adultos, sejam seus pais ou aqueles responsáveis pelos cuidados delas. Todos devem receber formação sobre prevenção de acidentes, como o das queimaduras. Percebeu-se durante o estudo, que o Enfermeiro está cotidianamente ligado ao papel educativo de promoção a saúde, mas que sem o apoio da equipe multiprofissional, essa missão torna-se algo não muito efetivo. Ressaltando a importância do trabalho conjunto, pois os pais precisam ser alertados quanto aos riscos, bem 
como intervir de maneira adequada. A elaboração de cartilhas, manuais e palestras também conseguem atingir esse público-alvo. Portanto, o preparo desses profissionais durante a graduação e a contínua atualização de conhecimentos, quanto às medidas de educação e saúde, são capazes de aprimorar a sua atuação nesse contexto.

\section{REFERÊNCIAS}

Batalha, Sara et al. Acidentes em Crianças e Jovens, Que Contexto e Que Abordagem? Experiência de Nove Meses no Serviço de Urgência num Hospital de Nível II. Acta Pediátrica Portuguesa, 47, p. 7-30, 2016.

Brito, Mychelangela de Assis et al. Fatores de risco no ambiente doméstico para quedas em crianças menores de cinco anos. Rev. Gaúcha Enferm, v. 38, n. 3, 2017.

Caricchio, Milena Braga Maia. Tratar brincando: o lúdico como recurso da fisioterapia pediátrica no Brasil. Revista Eletrônica Atualiza Saúde, v. 6, n. 6, p. 43-57, 2017.

Farias, Marcela Barbosa de et al. A experiência da criança hospitalizada com a realidade virtual em procedimentos dolorosos. Revista de Saúde Digital e Tecnologias Educacionais, v. 4, n. 2, 2019.

Lima, E. P. M. et al. Identificação dos conhecimentos de mãos na prevenção de acidentes domésticos com crianças da primeira infância. Enferm. Foco, v. 9, n. 4, p. 77-80, 2018.

Margotti, Edficher; Costa, Pedro Paulo Silva da; Corrêa, Allyson Maycon Chaves. A importância da prevenção de acidentes na infância: Um relato de experiência. Rev Enferm Atenção Saúde, v. 7, n. 1, p. 200-208, 2018.

Moita, Carina Estrela; Andrade, Alícia Maria Silva; Campos, Rita Cristina Gonçalves. Educação em saúde para prevenção de acidentes domésticos na infância. Revista de Trabalhos Acadêmicos, v. 1, n. 5, 2017. 
Passos, Daniele de Almeida; Santos, Walquiria Lene dos. O enfermeiro como educador para a prevenção dos principais acidentes ocorridos na primeira infância. Revista de Divulgação Científica Sena Aires, v. 5, n. 2, p. 124-35, 2016.

Sanches, Pedro Henrique Soubhia et al. Perfil epidemiológico de crianças atendidas em uma Unidade de Tratamento de Queimados no interior de São Paulo. Rev Bras Queimaduras, v. 15, n. 4, p. 246-250, 2016.

Scapin, Soliane et al. Realidade virtual no tratamento da dor em criança queimada: Relato de caso. Rev Bras de Queimaduras, v. 16, n. 1, p. 45-8, 2017.

Silva, Manalde Ferreira da et al. Determining factors of domestic accidents in early childhood. J Hum Growth Dev, v. 27, n. 1, p. 10-18, 2017. 